\title{
A co-created intervention with care home residents and university students following a service-learning methodology to reduce sedentary behaviour: The GET READY project protocol
}

\author{
Maria Giné-Garriga ${ }^{1,2}$, Marlene Sandlund ${ }^{3}$, Philippa M. Dall', Sebastien F.M. Chastin ${ }^{1,4}$, Susana Pérez², \\ Dawn A. Skelton' \\ 'School of Health and Life Sciences. Glasgow Caledonian University. Glasgow, United Kingdom; ${ }^{2}$ Department of Physical \\ Activity and Sport Sciences. Faculty of Psychology, Education and Sport Sciences (FPCEE) Blanquerna, Ramon Llull University. \\ Barcelona, Spain; ${ }^{3}$ Department of Community Medicine and Rehabilitation, Umeå University. Umeå, Sweden; ${ }^{4}$ Department of \\ Movement and Sport Science, Ghent University, Ghent, Belgium
}

All published work is licensed under Creative Common License CC BY-NC-SA 4.O (Attribution-NonCommercial-ShareAlike)

\begin{abstract}
Background: There is a growing demand for long-term care settings. Care-home residents are a vulnerable group with high levels of physical dependency and cognitive impairment. Long-term care facilities need to adapt and offer more effective and sustainable interventions to address older residents' complex physical and mental health needs. Despite the increasing emphasis on patient and public involvement, marginalised groups such as care-home residents, can be overlooked when including people in the research process. The GET READY project aims to integrate servicelearning methodology into Physical Therapy and Sport Sciences University degrees by offering students individual service opportunities with residential care homes, in order to co-create the best suited intervention with researchers, older adults of both genders (end-users) in care homes, health professionals, caregivers, relatives and policy makers. Methods: Stage 1 will integrate a service-learning methodology within a Physical Therapy module in Glasgow and Sport Sciences module in Barcelona, design two workshops for care home residents and one workshop for staff members, relatives and policy makers and conduct a co-creation procedure. Stage 2 will assess the feasibility, safety and preliminary effects of the co-created intervention in a group of 60 care home residents, within a two-armed pragmatic randomized clinical trial. ClinicalTrials.gov Identifier: NCT03505385.
\end{abstract}

Keywords: Co-creation, Care home residents, Physical activity, Sedentary behaviour, Service-learning methodology

\section{Introduction}

The number of older adults aged 65 or over in the population will increase significantly in the coming decades, with the proportion of those aged 80 years or above growing at a faster pace than any other age segment of the European Union's population'. This increase is likely to be linked to a growing demand for long-term care, placing a significant strain on health care resources. Care-home residents are a vulnerable group with high levels of physical dependency ${ }^{2}$, and with three-quarters having cognitive impairment ${ }^{3}$. This will require the adaptation of long-term care facilities' policies to offer more effective and sustainable interventions to address the complex physical and mental health needs of their residents ${ }^{4}$. It is well established that regular physical activity
(PA) limits the development and progression of chronic diseases and disabling conditions ${ }^{5}$. However, time spent in sedentary behaviour (SB) (defined as any waking behaviour

The authors have no conflict of interest. The present project had been funded by the European Commission Horizon 2020 Framework Programme (Reference: 747490 - GET READY).

Corresponding author:Maria Giné-Garriga, Glasgow Caledonian University: A267 Govan Mbeki Building, Cowcaddens Road, Glasgow G4 OBA. Scotland, United Kingdom

E-mail: Maria.GineGarriga@gcu.ac.uk

Edited by: Yannis Dionyssiotis

Accepted 7 August 2018 
characterized by an energy expenditure $\leq 1.5$ Metabolic Equivalent Tasks while in a sitting or reclining posture $)^{6}$ has increased substantially over the last three decades ${ }^{7}$ and increases with age ${ }^{8}$. SB has been gaining recognition as a risk factor, sometimes independent to PA status, for numerous health condition $s^{5}$ including cardiovascular disease ${ }^{9}$, type II diabetes ${ }^{10}$, obesity ${ }^{11}$, reduced mobility ${ }^{12}$ and poor functional performance, which can cause falls and fractures ${ }^{13}$. There is a large body of evidence which has focused on PA-based interventions to improve physical function and prevent falls in institutionalized older adults ${ }^{14}$. A meta-analysis confirmed that PA programmes could improve activities of daily living in older people living in long-term care facilities although the characteristics of the best intervention remain unclear ${ }^{15}$. However, there is still a gap in knowledge on how to change the mind set and activities offered to older residents by 'gatekeeper' health professionals and how to make 'moving more often' normal in the residential setting. Despite the growing interest in SB research, there has been a lack of studies focused on reducing SB in institutionalized older adults.

Patient and public involvement (PPI) has developed into an integral part of research practice over the last 25 years. Despite the increasing emphasis on PPI, marginalised groups, such as care-home residents, can be overlooked when including people in the research process, although a recent systematic review showed that older care-home residents could be successfully involved ${ }^{16}$. It is thought that involving end-users in the development of solution-based interventions using key elements derived from participatory methodologies such as PPI may increase the likelihood of producing sustainable change ${ }^{17}$. Emergent from the participatory design paradigm is a process called co-creation ${ }^{18}$, which is hypothesised to have a strong and enduring impact on health outcomes ${ }^{19}$, and may be a promising strategy to address other complex health behaviours. To our knowledge, there is only one previous study involving community-dwelling older adults to co-create an intervention to reduce $\mathrm{SB}^{20}$.

University degrees, such as Physical Therapy and Sport Sciences, need to have a practical approach rather than just be dominated by theory. Service learning is a teaching and learning strategy that involves students in community service, and integrates meaningful community service with instruction and reflection to enrich the learning experience, teach civic responsibility, and strengthen communities ${ }^{21}$. This project has the involvement of university students, care home residents (end-users), researchers, health professionals, care home staff, caregivers, family members and policy makers, enriching it with a distinctly multidisciplinary and inter-sectorial nature.

The GET READY project aims to integrate servicelearning methodology into Physical Therapy and Sport Sciences University degrees by offering students individual service opportunities with residential care homes ${ }^{22}$, in order to co-create the best suited intervention to reduce the SB of residents, with researchers, older adults of both genders (end-users) in care homes, health professionals, caregivers, family members and policy makers. The project will use a Participatory Action Research (PAR) methodology ${ }^{23}$ to support users to demand more responsive and integrated care programmes for chronic diseases, and to involve endusers in their health management and decision-making relevant to their own health ${ }^{24}$. This will then be tested in both a feasibility study, to estimate important parameters needed to design the main study, and a pilot study, to test the integrity of the study protocol for a definitive future trial, and to determine the acceptability of the intervention within that context ${ }^{25}$.

\section{Materials and methods}

The GET READY study will be divided into two stages. The first stage will focus on the integration of the servicelearning methodology within a University degree, and the design of three workshops for the co-creation of an intervention to reduce sedentary behaviour and enhance physical activity in care home residents. The second stage will focus on the feasibility of the intervention co-created in the previous stage, and a pilot study to consider preliminary effectiveness.

\section{Stage 1. Workshops and co-creation protocol}

The present study is being conducted in Glasgow (Scotland) and Barcelona (Catalonia). A purposive sampling strategy will be used to identify a total of 5 to 6 residents living in two care homes in Glasgow and 5 to 6 residents living in two care homes in Barcelona. The total sample will include residents of both genders, with different physical and cognitive conditions, physical activity levels, and amount of time spent in sedentary behaviour. Questions about current levels of physical activity, sedentary behaviour, walking ability, physical and cognitive function, will be explored with the care home staff to enable purposive sampling to take place, after inviting all residents to participate. The study has been approved by the Ethics Committee of the School of Health and Life Sciences of Glasgow Caledonian University, and the Ethics Committee of the Faculty of Psychology, Education and Sport Sciences Blanquerna (Ramon Llull University). All participants (or legal representatives if applicable) will sign an informed consent prior to participation.

Health professionals at the care homes will provide clinical and demographic information: gender, date of birth, marital status, medical conditions, medication, timespan in the care home, PA levels assessed with the International Physical Activity Questionnaire, and proxy report of self-report hours of sedentary time a day. This data will allow description of the study population.

The service-learning methodology will be integrated within a current module in the Physical Therapy degree in Glasgow Caledonian University and in the Sport Sciences degree in Blanquerna, Ramon Llull University in Barcelona, adding content related to service-learning methodology and 
successful experiences using service-learning methodology for undergraduate students, how to co-create a successful intervention, and tips on how to conduct a discussion group and foster positive group dynamics. A group of students will be involved in the design of two workshops (WS1 and WS2) for care home residents (as part of the module) including information regarding the health benefits of regular physical activity and reducing sedentary behaviour, barriers and motivators to be physically active and less sedentary, how to collect preferred strategies to be more physically active and less sedentary, as well as inform them about the importance of gathering end-users' opinion on how to design a "sit less move more" intervention for their daily living. Another group of students will design a workshop for staff members (WS3), relatives and policy makers to gather the same information.

Between WS1 and WS2, the care home residents will be asked to wear an ActivPAL ${ }^{\mathrm{TM}}$ monitor (PAL Technologies, Scotland), a valid "gold standard" method to measure postural sitting ${ }^{26,27}$, that is feasible for use with high adherence rates (>90\%) in older adults ${ }^{28}$. The monitor can record the total time the residents spend sitting, standing and walking. However, at very slow walking speeds (e.g. <0.5 $\mathrm{m} / \mathrm{s}$ ), walking may not be identified ${ }^{29}$, so we will use time spent upright (standing and walking) as our main outcome measure for physical activity, rather than differentiating between standing and walking ${ }^{30}$. We will also document time spent sitting or reclining - sedentary time. The monitor will be waterproofed, and will be attached to the front of the thigh by a researcher. Participants will wear the monitor continually (including overnight) for nine days, and the activPAL ${ }^{\mathrm{TM}}$ monitor will be removed by the researcher at a second meeting. A graphical representation of sedentary behaviour data will be fed back to participants at the second workshop, to raise awareness of their sedentary time and their sedentary behaviour patterns, to enable identification of the best-suited strategy to modify this behaviour as it may be that certain times of the day are more appropriate for intervention.

WS1 aims to inform care home residents about the health-related benefits of being physically active and less sedentary, and to gather relevant information regarding their PA and SB patterns and willingness to change behaviour. WS2 aims to use the activPAL ${ }^{\mathrm{TM}}$ data to explore their preferences and goals to enhance changes within their daily routines. Both workshops will be conducted by the same three to four students that had designed them with the 5 to 6 residents, with researcher supervision. The workshop for staff members (WS3) will be conducted by the same students that had designed it and the following participants will be invited: two researchers, three health professionals working in the care home (e.g. physical therapist, nurse, occupational therapist, geriatrician), one caregiver, one family member, and one policy maker. WS3 will be aimed at identifying PA and SB patterns of care home residents, their perceived limitations to enhance movement in care home residents, willingness to change and preferred strategies. WS 1 and WS2 will last no more than 60 minutes each and WS3 will last for no more than 90 minutes. Each workshop will be audio-taped and/or video-recorded with participants' consent. Topics for all groups will include: (a) acceptability, feasibility and preferences for particular intervention components within their daily lives coherent with their daily routine (safety, engagement), (b) personal goals and action planning, (c) barriers and motivators for PA and SB reduction, and (d) information on what may help people to reduce the amount of time spent sitting in their daily lives within the care home environment. We will include important theoretical constructs towards successful behaviour change such as education, goal setting and action planning, prompts, and embedding PA and SB reduction in to the daily routine.

\section{Stage 2. Feasibility and pilot study}

In stage 2 of the GET READY project we will assess the feasibility, safety and preliminary effects of the intervention co-created in the previous stage in a group of care home residents from the same care homes. Once the aforementioned three workshops have been analysed, a first draft of the intervention will be designed, shown to the participants of the WS, and amended accordingly following their comments and preferences.

It is proposed that there are eight general areas of focus addressed by feasibility studies ${ }^{31}$ : (a) acceptability, that looks at how the recipients, both targeted individuals and those involved in implementing programs, react to the intervention; (b) demand, that provides information on estimated use of selected intervention activities in a defined intervention population or setting; (c) implementation, that focuses on the extent, likelihood, and manner in which an intervention can be fully implemented as planned and proposed $^{32}$; (d) practicality, that explores the extent to which an intervention can be delivered when resources, time, commitment, or some combination thereof are constrained in some way; (e) adaptation, which focuses on changing program content or procedures, to be appropriate in new situations; ( $f$ ) integration, that assesses the level of system change needed to integrate a new program or process into an existing infrastructure or program; ( $\mathrm{g}$ ) expansion, which examines the potential success of an already-successful intervention with a different population or in a different setting; and (h) limited-efficacy testing, as many feasibility studies are designed to test an intervention in a limited way. Such tests may be conducted in a convenience sample, with intermediate rather than final outcomes, with shorter followup periods, or with limited statistical power.

To assess intervention feasibility, we will record recruitment and retention through the programme. We will conduct qualitative interviews with participants, caregivers, family members and care home staff at the end of the programme and interviews will be recorded and transcribed 
verbatim. Caregivers will be interviewed separately or together with the participant, depending on their preference. We will select for interview participants with low- and high-adherence. We will also seek to interview a sample of participants who discontinue the intervention. Using Thematic Analysis ${ }^{33}$, we will elicit participants' views on acceptability of the intervention and research procedures. Participant perspectives of their behaviour changes will be reported separately.

We will use mixed methods to assess the intervention feasibility and we will conduct a pilot study with stop and go decision points of a two armed pragmatic randomized clinical trial to assess the effectiveness of the co-created intervention on reducing SB and increasing $\mathrm{PA}$, in care home residents. This complex intervention co-created with care home residents and conducted by university students will be compared to usual care, conducted by health professionals working in the residential care homes.

\section{Participants of the feasibility and pilot studies}

Both countries will recruit approximately 30 care home residents from 2 homes (the duration of the intervention can't be anticipated as it depends on the co-creation process). Participants will be randomised to a PA group (usual care) or to the GET READY co-created intervention, in a cluster randomized pilot study. Eligible participants will be aged over 70 years willing to participate. Exclusion criteria will include comorbidity preventing participation (e.g. severe breathlessness, pain, or severe neurological disease), severe cognitive impairment, life expectancy of less than one year, being unable to rise out of a chair with help of one person, or being unlikely to undertake the intervention. Individual participants will discontinue participation if: they withdraw consent or no longer wish to take part; the health professional overseeing their care decides the participant is no longer able to take part; otherwise at the discretion of the investigator (e.g. risk to safety of staff). We will collect outcome data from those who withdraw, if they are willing.

\section{Randomisation}

The two care homes will be randomised after baseline measures, using an independent and secure, web-based randomisation procedure. The randomisation system will be maintained by a statistician independent of the analysis and research teams to ensure blinding of allocation and analysis.

\section{Data collection}

Measures will be assessed at baseline and end of intervention. Recruitment, retention and adherence rates will be used to assess feasibility ${ }^{34}$ and, alongside effect size, will be used to refine the sample size required for a future definitive trial. The acceptability of the intervention will be assessed using two approaches: 1) all participants (or their caregivers in the case of cognitive impairment) will be asked to rate their level of satisfaction with the intervention on a 5-point Likert-type scale, as used in a previous PA intervention ${ }^{35}$; 2) a group of participants will be convened to explore their views of how the programme can be improved.

Primary outcome: Sedentary behaviour as total sitting time, breaks in sitting time and the a ratio (ratio among breaks and transitions), as measured by the gold standard activPAL ${ }^{\mathrm{TM}}$ monitor (PAL Technologies, Scotland) ${ }^{29}$. Secondary outcomes: (a) physical activity as time spent upright (same device) ${ }^{29}$, (b) physical function: 5-chair rise test and gait speed ${ }^{36}$. And anticipated secondary outcomes will include: activities of daily living; adherence; selfrated health and health-related quality of life; anxiety and depressive symptoms; social network; physical activity selfregulation or self-efficacy for exercise; fatigability; fear of falling; cognition and executive function. This list may vary following discussion with residents and care home staff about meaningful patient centred outcomes and considering participant and carer burden.

\section{Data analysis}

For efficacy analysis, descriptive statistics (e.g. means, medians, proportions, standard deviations and ranges) of baseline demographic and health status data will be calculated for both trial arms. We will compare changes in primary and secondary outcomes between the two trial arms, using an analysis of covariance to adjust for the baseline score and stratifying variables. Intervention effects will be represented by point estimates ( $95 \%$ confidence intervals). Statistical analysis will be performed to look at power for future sample size estimates.

For qualitative analysis of the workshops (stage 1), transcripts will be analysed using Thematic Analysis ${ }^{33}$ with embedded constant comparative method. Analysis will be facilitated by the use of text management software (NVivo). Two researchers will be involved in the analysis to ensure reliability of interpretation and coding. Rigour will be ensured in a number of ways. Firstly, the constant comparative method relies on the researcher seeking disconfirming evidence for developing theory. This ensures transcripts are not analysed selectively, and that the totality of the dataset is consistent with the reported findings. Secondly, two members of the research team with qualitative research experience will meet regularly with the researcher to discuss emerging codes and categories, the interpretation of key texts and potential new lines of enquiry. Finally, towards the end of the analysis, once concepts, properties and dimensions have been formulated; two independent researchers will examine transcripts and assign established codes. A comparison of coding will be carried out to assess agreement between coders.

\section{Discussion}

This is, to our knowledge, the first study involving University students using a service-learning methodology in care home settings to co-create an intervention with care home residents aimed at decreasing sedentary 
behaviour and increasing physical activity levels.

The co-creators aim to develop an intervention which is tailored to end-users' preferences and is congruent with their daily life. Physical activity and sedentary behaviour are ubiquitous throughout the $\mathrm{day}^{37}$, and recommendations show that interventions need to modify the daily routine $e^{38,39}$ and be integrated within older adult's everyday activities, to be able to achieve sustainable changes in behaviour. Breaking up of sedentary behaviour, through standing up approximately once an hour, without any change in total sitting time or activity levels, have been shown to improve physical function over a 10 week period in residents of sheltered housing ${ }^{40}$. However, this involved the use of a vibrating prompt and considerable time in motivational interviewing, which may not be possible in the care home setting.

There is a pressing need for interventions to increase activity and enhance independence among care home residents. This is to enable people to 'move more and sit less', live better lives, and to mitigate the increasing burden on health and social care of care home residents-related dependency. Fall-related injury and activity restriction are often responsible for deterioration in quality of life in carehome residents.

Care homes are unique research settings and care-home residents need specific consideration in relation to how they may be involved in research. A recent systematic review identified multiple barriers to and facilitators of involving residents as PPI members grouped under: social factors, skills, resources, care-home organisational factors and the organisation of the research ${ }^{16}$. These factors will be anticipated in the present project during the workshop.

Curricula of health professionals should comprise lecturebased and experiential training on population medicine, health promotion/disease prevention and social determinants of health ${ }^{41}$. PPI and service-learning are unique approaches to train Physical Therapy and Sport Science students how to provide patient care from a population perspective. Servicelearning is a structured experience that combines community service with specific learning objectives, preparation and reflection through community-academic partnerships ${ }^{42}$. Studies demonstrate that, with appropriate supervision, student involvement not only maintains or improves patient satisfaction outcomes, but can contribute to improving patient safety and health outcomes ${ }^{43,44}$.

The main objectives of the GET READY project are wider than improving quality of life and physical function in residents, as we aim to raise awareness, knowledge, skills and passion of graduates entering the workforce, and to lead to a change in culture of professionals working with this frail and co-morbid population. The GET READY intervention will be conducted in Glasgow and Barcelona, with the end point being a culturally and gender wide sustainable program ready to be tested in a RCT in different locations around Europe in a future research proposal.

\section{Acknowledgements}

The authors wish to acknowledge the staff and residents of the Erskine Glasgow Home and Orchard Grove Residential Care Home in Glasgow, and of the Parc Sanitari Pere Virgili and the Association of Alzheimer Disease in Barcelona for their collaboration. We also thank the collaboration of the students from Glasgow Caledonian University (Glasgow) and the Faculty of Psychology, Education and Sport Sciences Blanquerna (Barcelona) that collaborated.

\section{References}

1. European Union Eurostat. Population structure and ageing Luxembourg: 2017. Available at: http://ec.europa.eu/eurostat/ statisticsexplained/index.php/Population_structure_and_ageing.

2. Gordon AL, Franklin M, Bradshaw L, et al. Health status of UK care home residents: a cohort study. Age Ageing 2013;43:97-103.

3. Lievesley N, Crosby G, Bowman C, et al. The Changing Role of Care Homes. London: Bupa and Centre for Policy on Ageing, 2011. Available at: http://www.cpa.org.uk/information/reviews/ changingroleofcarehomes.pdf

4. Lowton K, Laybourne A, Whiting D, et al. High impact actions: preventing falls and encouraging exercise. Nurs Manag (Harrow) 2010; $17(4): 22-5$

5. World Health Organization. Global recommendations on physical activity for health. Geneva: World Health Organization, 2010. Available at: http://apps.who.int/iris/bitstream/handle/ 10665/44399/9789241599979_eng.pdf?sequence=1

6. Sedentary Behaviour Research Network. Letter to the editor: standardized use of the terms "sedentary" and "sedentary behaviours". Appl Physiol Nutr Metab 2012;37:1-3.

7. Van der Ploeg HP, Venugopal K, Chau JY, et al. Non-occupational sedentary behaviors: population changes in The Netherlands, 19752005. Am J Prev Med 2013;44(4):382-7.

8. Bellettiere J, Carlson JA, Rosenberg D, et al. Gender and Age Differences in Hourly and Daily Patterns of Sedentary Time in Older Adults Living in Retirement Communities. PLoS One 2015; 10(8):e0136161

9. Katzmarzyk PT, Church TS, Craig CL, et al. Sitting time and mortality from all causes, cardiovascular disease, and cancer. Med Sci Sports Exerc 2009; 41(5):998-1005.

10. Wilmot EG, Edwardson CL, Achana FA, et al. Sedentary time in adults and the association with diabetes, cardiovascular disease and death: systematic review and meta-analysis. Diabetologia 2012;55:2895-905

11. Jakes RW, Day NE, Khaw KT, et al. Television viewing and low participation in vigorous recreation are independently associated with obesity and markers of cardiovascular disease risk: EPIC-Norfolk population-based study. Eur J Clin Nutr 2003;57(9): 1089-96.

12. Nelson ME, Rejeski WJ, Blair SN, et al. Physical activity and public health in older adults: Recommendation from the American College of Sports Medicine and the American Heart Association. Med Sci Sports Exerc 2007;39:1435e 1445 .

13. Gianoudis J, Bailey CA, Daly RM. Associations between sedentary behaviour and body composition, muscle function and sarcopenia in community-dwelling older adults. Osteoporos Int 2015; 26(2):571-9.

14. Weening-Dijksterhuis E, de Greef MH, Scherder EJ, et al. Frail institutionalized older persons: A comprehensive review on physical exercise, physical fitness, activities of daily living, and quality-of-life. 
Am J Phys Med Rehabil 2011 ;90(2): 156-68.

15. Crocker T, Young J, Forster A, et al. The effect of physical rehabilitation on activities of daily living in older residents of longterm care facilities: Systematic review with meta-analysis. Age Ageing 2013;42:682e688.

16. Backhouse T, Kenkmann A, Lane K, et al. Older care-home residents as collaborators or advisors in research: a systematic review. Age Ageing 2016;45(3):337-45

17. Green LW, O'Neill M, Westphal M, et al. The challenges of participatory action research for health promotion. Promot Educ 1996;3(4):3-5.

18. Sanders EBN, Stappers PJ. Co-creation and the new landscapes of design. CoDesign 2008;4(1):5-18

19. Greenhalgh T, Jackson C, Shaw S, et al. Achieving research impact through co-creation in community-based health services: Literature review and case study. Milbank Q 2016:94(2):392-429.

20. Leask CF, Sandlund M, Skelton DA, et al. Co-creating a tailored public health intervention to reduce older adults' sedentary behaviour. Health Educ J 2017;76(5):595-608.

21. Heffernan K. Excerpted from: Fundamentals of Service-Learning Course Construction. Boston: Campus Compact Publisher, 2001, pp 2-7.

22. Laks J, Wilson LA, Khandelwal C, et al. Service-Learning in Communities of Elders (SLICE): Development and Evaluation of an Introductory Geriatrics Course for Medical Students. Teach Learn Med 2016:28(2):210-8

23. Baum F, MacDougall C, Smith D. Participatory action research. J Epidemiol Community Health 2006;60(10):854-7.

24. Tsartsara S, Kivilehto A (coordinators). B3 Action Group on Replicating and Tutoring Integrated Care for Chronic Diseases. EIP AHA, 2015. Luxembourg: Publications Office of the European Union. Available at: 509ce7 1a5bbb3_b3_action_plan.pdf

25. Lancaster GA, Dodd S, Williamson PR. Design and analysis of pilot studies: recommendations for good practice. J Eval Clin Pract 2004; 10(2):307-12.

26. Kozey-Keadle S, Libertine A, Lyden K, et al. Validation of wearable monitors for assessing sedentary behavior. Med Sci Sports Exerc 2011;43(8):1561-7.

27. Sellers C, Dall PM, Grant PM, et al. Validity and reliability of the activPAL 3 for measuring posture and stepping in adults and young people. Gait Posture 2016;43:42-7.

28. Dall PM, Skelton DA, Dontje ML, et al. Characteristics of a protocol to collect objective physical activity/sedentary behaviour data in a large study: Seniors USP (understanding sedentary patterns). J Meas Phys Behav 2018;1(1):26-31

29. Chastin SF, Granat MH. Methods for objective measure, quantification and analysis of sedentary behaviour and inactivity. Gait Posture 2010;31(1):82-6.
30. Grant PM, Granat MH, Thow MK, Maclaren WM. Analyzing freeliving physical activity of older adults in different environments using body-worn activity monitors. J Aging Phys Act 2010; 18(2):171-84.

31. Bowen DJ, Kreuter M, Spring B, et al. How we design feasibility studies. Am J Prev Med 2009;36(5):452-57.

32. Green L, Glasgow RE. Public health asks of systems science: to advance our evidence-based practice, can you help us get more practice based evidence? Am J Public Health 2006;96:406-9.

33. Braun V, Clarke V. Using thematic analysis in psychology. Qual Res Psychol 2006;3(2):77-101

34. Hawley-Hague H, Horne M, Skelton DA, et al. Review of how we should define (and measure) adherence in studies examining older adults' participation in exercise classes. BMJ Open 2016;6:eO 11560.

35. Hunter RF, Tully MA, Davis M, et al. Physical activity loyalty cards for behavior change: a quasi-experimental study. Am J Prev Med 2013;45(1):56-63.

36. Guralnik JM, Ferrucci L, Simonsick EM, et al. Lower-extremity function in persons over the age of 70 years as a predictor of subsequent disability. N Engl J Med 1995;332(9):556-61.

37. Sartini C, Wannamethee SG, lliffe S, et al. Diurnal patterns of objectively measured physical activity and sedentary behaviour in older men. BMC Public Health 2015; 15(1):609.

38. Chastin SFM, Fitzpatrick N, Andrews M, et al. Determinants of sedentary behavior, motivation, barriers and strategies to reduce sitting time in older women: A qualitative investigation. Int J Environ Res Public Health 2014; 1 1(1):773-91.

39. Greenwood-Hickman MA, Renz A, Rosenberg DE. Motivators and barriers to reducing sedentary behavior among overweight and obese older adults. Gerontologist 2016;56:660-8.

40. Harvey JA, Chastin SFM, Skelton DA. Breaking sedentary behaviour has the potential to increase/ maintain function in frail older adults. JFSF 2018;3(1):26-34.

41. Meyer SM, Garr DR, Evans C, et al. Advancing interprofessional clinical prevention and population health education a curriculum development quide for health professions faculty. 2016. Available at: http://www. teachpopulationhealth.org/uploads/2/1/9/6/21964692/ipe_ crosswalk_2016_update.pdf

42. Stewart T, Wubbena ZC. A systematic review of service-learning in medical education: 1998-2012. Teach Learn Med 2015; 27:115-22

43. Seiden SC, Galvan C, Lamm R. Role of medical students in preventing patient harm and enhancing patient safety. Qual Saf Health Care 2006; 15(4):272-6.

44. Simon SR, Peters AS, Christiansen CL, et al. The effect of medical student teaching on patient satisfaction in a managed care setting. J Gen Intern Med 2000; 15(7):457-61. 